\title{
Compressing IP Forwarding Tables with Small Bounded Update Time
}

\author{
Yuanyuan Zhang ${ }^{\mathrm{a}}$, Mingwei Xu ${ }^{\mathrm{a}, *}$, Ning Wang ${ }^{\mathrm{b}}, \mathrm{Jun} \mathrm{Li}^{\mathrm{c}}$, Penghan Chen ${ }^{\mathrm{d}}$, Fei \\ Liang ${ }^{\mathrm{d}}$ \\ ${ }^{a}$ Tsinghua National Laboratory for Information Science and Technology, \\ Department of Computer Science and Technology, Tsinghua University, China \\ ${ }^{b}$ Institute for Communication Systems, University of Surrey, United Kingdom \\ ${ }^{c}$ Department of Computer and Information Science, University of Oregon, United States \\ ${ }^{d}$ Broadband Network Research Center, Beijing University of Posts and Telecommunications, \\ China
}

\begin{abstract}
With the fast development of the Internet, the size of Forwarding Information Base (FIB) maintained at backbone routers is experiencing an exponential growth, making the storage support and lookup process of FIBs a severe challenge. One effective way to address the challenge is FIB compression, and various solutions have been proposed in the literature. The main shortcoming of FIB compression is the overhead of updating the compressed FIB when routing update messages arrive. Only when the update time of FIB compression algorithms is small bounded can the probability of packet loss incurred by FIB compression operations during update be completely avoided. However, no prior FIB compression algorithm can achieve small bounded worst case update time, and hence a mature solution with complete avoidance of packet loss is still yet to be identified. To address this issue, we propose the Unite and Split (US) compression algorithm to enable fast update with controlled worst case update time. Further, we use the US algorithm to improve the performance of a number of classic software and hardware lookup algorithms. Simulation results show that the average update speed of the US algorithm is a little faster
\end{abstract}

\footnotetext{
${ }^{*}$ Corresponding author: Mingwei Xu; Phone, 0086-(0)10-62781572; Email Address, xmw@cernet.edu.cn

Email address: zhyyuan1019@gmail.com (Yuanyuan Zhang)
}

Preprint submitted to Computer Networks

June 8, 2016 
than that of the binary trie without any compression, while prior compression algorithms inevitably seriously degrade the update performance. After applying the US algorithm, the evaluated lookup algorithms exhibit significantly smaller on-chip memory consumption with little additional update overhead.

Keywords: FIB Compression, FIB update, IP address lookup, tries, longest prefix matching

\section{Introduction}

\subsection{Background and Motivation}

The size of Forwarding Information Bases (FIBs) of backbone routers in the Internet has been increasing by around $15 \%$ every year [1. The FIB (DFZ 5 entries) size exceeded $512 \mathrm{~K}$ on August 13th in 2014, exceeding the hardware capacity of many legacy Cisco routers [2]. In addition, there were VPN routes which could be as many as the DFZ entries. As a result, it took about a week for these routers to upgrade their hardware capacity, and it has already been observed that the web browsing and content downloading speed was slowed down during the period. In the literature, technical schemes have already been proposed to solve such a problem, and among them FIB compression is a promising way to alleviate the growth pressure of FIBs in the Internet.

In fact, even if the FIB size does not exceed the capacity of routers, FIB compression is still beneficial for IP lookup. Generally, there are two kinds of IP lookup solutions. The first kind is hardware-based solutions, such as TCAMbased solutions [3, 4, 5, 6] and FPGA-based solutions [7, 8, 9, 10. For this kind of IP lookup solutions, compressing the FIBs can significantly save hardware cost and power consumption. The second kind is software-based solutions, such as 11, 12, 13, 14. For this kind of IP lookup solutions, compressing the FIBs reduces the probability of cache misses, and thereby achieves faster lookup speed.

As mentioned in BS [15], EAR [16], and FIFA [17, packet loss may happen 
when the compression or update algorithm is too slow. The FIB after compression is stored and looked up in the data plane of a router. When a FIB update message arrives, the router has to suspend the lookup process and buffer the incoming packets in a queue. The queue can only buffer finite packets, thus the update of the compressed FIB should be as fast as possible. If the update time is not small enough in the worst case, the buffer in the data plane may overflow and packet loss may happen. This is the main reason why vendors and ISPs are not willing to adopt FIB compression algorithms in real routers. Therefore, this paper targets at a practical FIB compression algorithm with small bounded update time.

\subsection{State-of-the-art and their Limitations}

Due to the significance of FIB compression, various compression solutions have been proposed, such as ORTC [18] and its successors [19, 20, 17, auto aggregation [21, 4-level 22], entropy compression [23, EAR[16], and NSFIB compression 24], etc. Among them, ORTC constructs the optimal FIBs in terms of the number of prefixes. Entropy compression pursues the optimal compression algorithm in terms of information entropy, but the compression results are no longer in the prefix format, thus cannot cooperate with existing IP lookup algorithms. NSFIB is an aggressive compression method which can exceed the optimal compression ratio of ORTC at the cost of changing the forwarding behavior. Although some classic compression algorithms (such as SMALTA [19], EAR, 4-level, auto aggregation, etc.) claimed to support fast

45 update, no prior algorithm is able to achieve small bounded worst case update time. Only when the worst case of update time is small bounded, the risk of packet loss during update can be fundamentally avoided.

\subsection{Proposed Solution Overview}

In this paper, we propose the Unite and Split (US) compression algorith-

${ }_{50} \mathrm{~m}$. The top level strategy of conventional FIB compression algorithms is to 
either make the best effort for compression ratio or to identify a trade-off between compression ratio and update speed, but it should be noted that no prior compression algorithm has a reasonable worst case bound of update time. In contrast, the objective of our US algorithm is to make the best effort for compression ratio in the premise of small bounded update time.

We use a trie $\rrbracket^{1}$ structure to illustrate the key compression technique of our proposed US algorithm. As shown in the first trie of Figure 2(a), it has three nodes with non-empty next hops: $q$ and its two child nodes $q_{1}$ and $q_{2}$. According to the longest prefix matching rule, an IP address either matches $q_{1}$ or $q_{2}$. In other words, there are at most two lookup results for any incoming IP packet. Therefore, we can replace this trie by one node with two next hops. In other words, the two child nodes $q_{1}$ and $q_{2}$ can be compressed (united) into their parent node $q$ with two next hops, where the left next hop belongs to $q_{1}$, and the right next hop belongs to $q_{2}$. Similarly, when two of the three nodes have nonempty next hops (the middle three tries in Figure 2(a), they can be compressed into one node with two next hops. However, we do not always perform such compression because when only one of the three nodes has a next hop (such as the three tries in Figure 2(b)], such compression does not reduce the number of prefix nodes 2 , but brings additional update overhead. In this case, we split 70 the prefix node to guarantee that every node has either two next hops or none for the sake of storage and lookup efficiency. For each trie node, we use the variable oldport to store the next hop before compression for the sake of correct update, and use variables leftport and rightport to store the left next hop and right next hop after compression respectively.

The US algorithm consists of two kinds of operations: unite and split, and it traverses the trie twice. In the first postorder traversal of the trie, we conduct the unite operations to reduce the number of prefix nodes. In the second postorder traversal of the trie, we conduct split operations on those nodes which do not

\footnotetext{
${ }^{1}$ Trie is a classic data structure to represent a FIB.

${ }^{2}$ Prefix nodes refer to the trie nodes with next hops.
} 
participate in the unite operations. To bound the update time, it is guaranteed that every trie node participates in at most one unite operation, and the nodes modified by each unite operation are confined in two adjacent levels. In this way, at most 3 trie nodes need to be updated by any update message. The main advantage of our algorithm is that the update speed is fast and the worst case of update time is small bounded. Simulations using real-world FIBs (around 512K entries) and updates on CPU platform with Intel(R) Core i7-3517U 1.9GHz \& $2.4 \mathrm{GHz}$ and $8 \mathrm{~GB}$ RAM show that the update speed of US ranges from 2.16 Mups (Million updates per second) to 107.75 Mups with a mean of 18.64 Mups, while the industry standard is only 100 Kups.

The cost of US is an additional step for the lookup. After looking up the 90 $\quad$ FIB compressed by US, suppose the length of the matched prefix is $n$, we check the $n+1^{t h}$ bit in the incoming IP address: if it is 0 , we report the leftport; if it is 1 , we report the rightport.

US can work perfectly with existing FIB compression algorithms and IP lookup algorithms. Simulation results show that about $7 \%$ of prefixes can be reduced when applying US to the optimal compression algorithm ORTC. US alone can not compete with ORTC in terms of compression, but the combination of US and ORTC can beat ORTC in compression (but the combination of US and ORTC can not be updated easily). Around 35\% on-chip memory can be saved when applying US to existing well-known IP lookup algorithms. Although some conventional compression algorithms can also be applied to existing IP lookup algorithms, the negative effect is that the update overhead will be aggravated significantly after compression. In contrast, since the worst case of US update is small bounded, the update complexity after US compression stays the same as that before US compression.

\subsection{Key Novelties}

FIB compression is a well studied field, and there have been various solutions in the literature. It seems there is very limited room for further improvements. Conventional FIB compression algorithms compress the prefix nodes with the 
same next hops, and each prefix node is still related to one next hop after compression. In contrast, we find a new way to compress the number of prefixes by allowing that each prefix node is related to two next hops. Specifically, we change the conventional "one next hop per prefix node" structure into a "two next hops per prefix node" structure. In other words, in our algorithm, the prefix and next hop information of child nodes are united to their parent node.

With regard to update, given an update message, conventional compression algorithms often compress the sub-trie rooted at the updating node, and the worst case is to re-compress the whole trie. In the US algorithm, we constrain the unite operations in two adjacent levels of the trie. Thus, given any update message, the worst case is to update three nodes.

Paper organization: the rest of the paper is organized as follows. Section 2 introduces our proposed US algorithm. Section 3 describes the update and lookup algorithm of US. Section 4 shows the application of US to FIB compression and IP lookup algorithms. Section 5 evaluates the performance of US. Section 6 discusses the related work. Finally, Section 7 concludes the paper.

\section{Proposed Solution}

\subsection{Background}

FIB, trie and nodes: Given an incoming packet, the Forwarding Information Base (FIB) is searched to decide which egress port (i.e., next hop) the packet should be forwarded to. Each FIB entry includes at least two fields: prefix and next hop. Binary trie 25 is a classic data structure to store a FIB. Each FIB entry is represented by a prefix node in the trie. The path from the root node to the prefix node corresponds to the prefix, and the corresponding next hop is stored in the prefix node. We call a node without a next hop an empty node. We define the level of a node as its hop-count distance to the root node whose level is 0 . The level of a prefix node is equal to the length of the corresponding prefix. The nodes without child nodes are called leaf nodes, while others are called internal nodes. 


\subsection{Rationale}

There are mainly three metrics for FIB compression algorithms: compression

140 ratio in terms of number of prefix nodes at the cost of complicated update and long compression time. As mentioned above, when handling the updates, the lookup process is forced to be suspended, and the incoming packets are buffered. Only if the worst case of update time is small bounded, the risk of packet loss can

\footnotetext{
${ }^{3}$ Next hop similarity means that in a small sub-trie, many prefix nodes share the same next hops.
} 
the pre-pocessor depends on next hop similarity, the compression performance will vary in different scenarios (i.e., higher next hop similarity leads to better compression ratio).

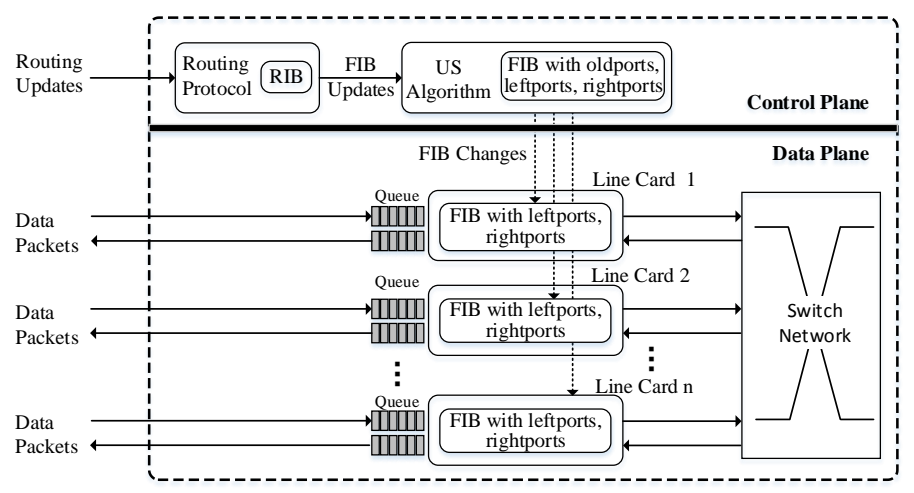

Figure 1: Router architecture.

\subsection{Router Architecture}

Before going to the details of the US algorithm, we first show how it operates in a real router. Figure 1 shows the architecture of a router. It consists of the control plane and the data plane. The control plane stores the RIB (Routing Information Base) containing all IP routing information. The prefixes and their selected next hops (a subset of the RIB) constitute the original FIB. Then the original FIB is compressed by US into a compressed FIB which is stored in the control plane. For the sake of fast update, the compressed FIB contains full information (i.e., oldports, leftports, and rightports). In the data plane, each line card has one copy of the compressed FIB which only contains leftports and rightports. Given an incoming packet, the line card looks up the FIB, gets a next hop, and then forwards it through the switch network. Each line card has a queue to buffer the incoming data packets.

As shown in Figure 1, when an update message arrives, first the RIB stored in the control plane will be updated by the routing protocol. If this leads to any FIB update, the update algorithm of US will be applied to the compressed

FIB containing full information stored in the control plane. This will result in 
changes of several prefix trie nodes. These changes are installed in the FIBs (only with necessary information for lookup) stored in the line cards of the data plane. During the installation process, the incoming data packets are buffered in the queue and cannot be forwarded. If there are too many prefix changes to handle per routing update, the FIBs in the line cards cannot be updated fast enough. In this case, the queues may overflow and packet loss may happen. The superiority of US lies in that: it can make the FIB in the line card as small as possible, while ensures fast update speed with controlled worst case update time.

\subsection{Unite and Split Algorithm}

The US algorithm traverses the trie two times. First US traverses the trie in postorder and performs unite operations according to four unite models. After the first traversal, there are two kinds of prefix nodes: the nodes with two next hops and the nodes with only one next hop. The nodes with one next hop will be split in the second traversal. We present the details of the four unite models and the split operation as follows.

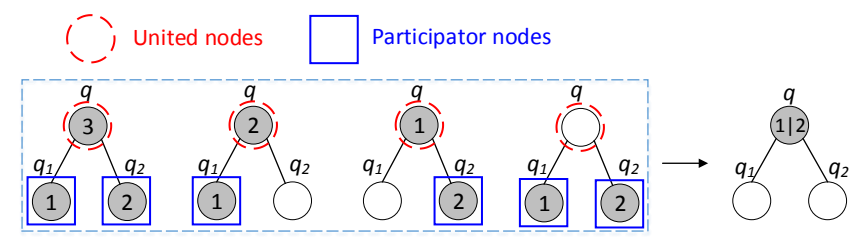

(a) Unite models

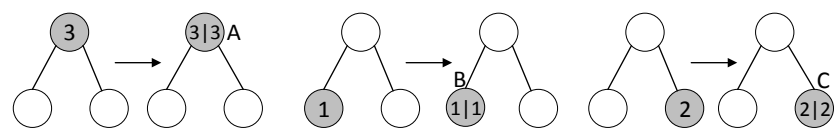

(b) Split models. Node A, B, and C are split nodes.

Figure 2: The models of the US algorithm. 


\subsubsection{Unite Models}

As shown in Figure 2(a), given a node $q$, its left child node $q_{1}$ and its right child node $q_{2}$, there are four unite models. First, $q, q_{1}$ and $q_{2}$ all have next hops. Second, only $q$ and $q_{1}$ have next hops. Third, only $q$ and $q_{2}$ have next hops. Fourth, only $q_{1}$ and $q_{2}$ have next hops. These four models are united into the same result: $q_{1}$ and $q_{2}$ have no next hops, and $q$ has two next hops: $1 \mid 2$, where " 1 " is the next hop of $q_{1}$ (or $q$ ), and "2" is the next hop of $q_{2}$ (or $q)$. After the unite operations, the nodes with two next hops are called united nodes, and the nodes that participate in the unite operations other than the united nodes are called participator nodes. In Figure 2(a), the nodes marked with dashed circles are united nodes, and the nodes marked with squares are participator nodes. To control the update time, every trie node can participate in at most one unite operation, and the nodes modified by each unite operation are confined in two adjacent levels.

\subsubsection{Split Operation}

As aforementioned, there are two kinds of prefix nodes after the first traversal: united nodes and nodes with only one next hop. In the second traversal, we conduct split operations on the nodes with only one next hop. The split operation is very simple: just change the next hop $h$ into two next hops $h \mid h$. The prefix nodes that are split are called split nodes. In Figure 2(b), we show three cases of the split operations. Node A, B, and C are split nodes.

In summary, US performs unite operations in the first traversal, and performs split operations in the second traversal. The pseudo codes of the unite and split operations are shown in Algorithm 1 and Algorithm 2, respectively.

\subsubsection{Example}

We now give an example of the US algorithm in Figure 3. It shows the original trie and the trie after US compression. Specifically, during the first traversal, node $\mathrm{F}$ and $\mathrm{G}$ are united into node $\mathrm{D}$ with two next hops 1|4; node $\mathrm{H}$ 


\begin{tabular}{l}
\hline Algorithm 1: Unite(TrieNode $\left.{ }^{*} \mathbf{q}\right)$ \\
\hline $\mathbf{1}$ if $q$ is NULL then \\
$\mathbf{2} \quad$ return; \\
$\mathbf{3} q_{1}=\mathrm{q} \rightarrow \mathrm{left} ;$ \\
$\mathbf{4} q_{2}=\mathrm{q} \rightarrow \mathrm{right;}$ \\
$\mathbf{5}$ Unite $\left(q_{1}\right) ;$ \\
$\mathbf{6}$ Unite $\left(q_{2}\right) ;$ \\
$\mathbf{7}$ if two or three nodes of $q, q_{1}, q_{2}$ have next hops and are not united nodes \\
then \\
$\mathbf{8} \quad$ perform the unite operation according to the four unite models; \\
\hline
\end{tabular}

\begin{tabular}{l}
\hline Algorithm 2: Split(TrieNode $\left.{ }^{*} q\right)$ \\
\hline $\mathbf{1}$ if $q$ is NULL then \\
$\mathbf{2} \quad$ return; \\
3 Split $(q \rightarrow$ left); \\
4 Split $(q \rightarrow$ right); \\
$\mathbf{5}$ if $q$ is a prefix node with only one next hop then \\
$\mathbf{6} \quad$ split the next hop of $q ;$ \\
\hline
\end{tabular}


into node A with two next hops 2|1. After the first traversal, node $\mathrm{K}$ with next hop 1 is left behind. During the second traversal, the next hop of node $\mathrm{K}$ is split. As a result, node $\mathrm{K}$ has two next hops $1 \mid 1$. In this example, the number of prefixes is reduced from 8 to 4 after US compression.

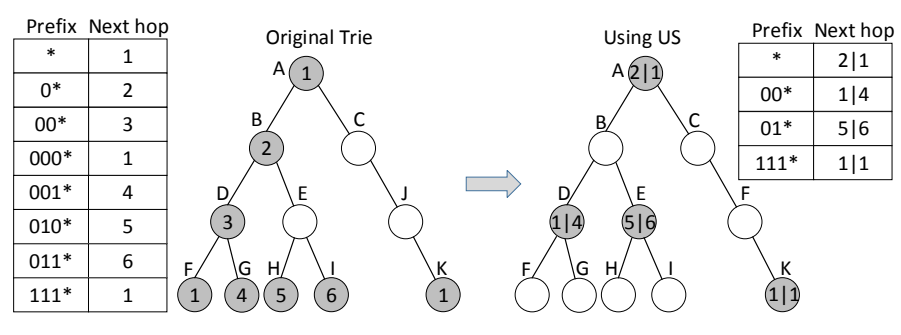

(a) The orignal Trie and FIB

(b) the Trie and FIB after compressed by US

Figure 3: An example of US.

$$
\text { de }
$$

their oldports are not empty. The whole trie structure with oldports is kept in the control plane so that we can know exactly what the trie before compression looks like when handling an update message. In the data plane, we only store

${ }_{240}$ the prefixes with leftports and rightports, while do not store the empty leaf nodes. In other words, we reduce the usage of fast memory in the data plane at the cost of more usage of slow memory in the control plane.

Actually, our US algorithm can compress the FIBs without tries. One straightforward way is to sort the prefixes, and then do compression for the 245 adjacent prefixes. However, this method will incur complicated update.

\section{Update and Lookup Algorithm of US}

\subsection{Update Algorithm of US}

There are two kinds of update messages: announcement and withdrawal. Given an announcement message: [announce $p: h$ ], it means that we should either insert a new prefix $p$ with next hop $h$ or change the next hop of the existing prefix $p$ to $h$. Given a withdrawal message: [withdraw $p$ ], it means 
we should delete the prefix $p$ and its corresponding next hop. In practice, the update operations are usually performed in the trie. In the following, we discuss the incremental update algorithm of US for the announcement and withdrawal messages separately.

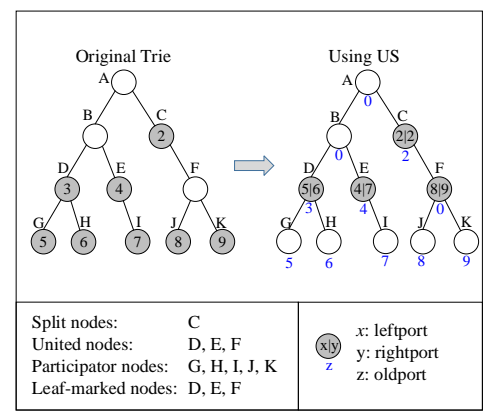

\begin{tabular}{|c|c|c|}
\hline & [announcement] 4 cases & [withdrawal] 3 cases \\
\hline Case I: united nodes. & [announce $01 *: 1] \quad \mathrm{E}(117)$ & [withdraw 01*] \\
\hline Case II: participator nodes. & [announce $\left.000^{*}: 1\right]$ & [withdraw $000 *$ ] \\
\hline Case III: split nodes. & [announce $1 *: 1]$ & [withdraw 1*] \\
\hline Case IV: empty/new nodes. & [announce $0 *: 1]$ & \\
\hline
\end{tabular}

Figure 4: An example for US update. $\mathrm{G}$ and $\mathrm{H}$ are united into $\mathrm{D}, \mathrm{I}$ is united into E, J and $\mathrm{K}$ are united into F, C is split. For the sake of fast update, we do not delete G, H, I, J, and K. For the sake of fast lookup, we mark node D, E and $\mathrm{F}$ as leaf nodes. For each update message, we do not show the whole trie after update, but only show the changed part in the right table.

\subsubsection{Announcement Handling}

To support update, we define three kinds of ports for every trie node: oldport, leftport, and rightport, where oldport refers to the next hop before compression, leftport and rightport are the two next hops after compression. The update algorithm for an announcement message [announce $p: h$ ] proceeds in two steps: first, we set the oldport of $n_{p}$ to $h$, where $n_{p}$ refers to the corresponding trie node of prefix $p$; second, we update the leftport and rightport fields according to the node type (united nodes, split nodes, etc.) of $n_{p}$. Specifically, there are four cases as follows.

- Case I: $n_{p}$ is a united node. There are three situations: 1) when the two child nodes of $n_{p}$ are both participator nodes, the leftport and rightport of $n_{p}$ keep unchanged; 2) when only the left child of $n_{p}$ is a participator 
node, the rightport of $n_{p}$ is changed to $\left.h ; 3\right)$ when only the right child of $n_{p}$ is a participator node, the leftport of $n_{p}$ is changed to $h$.

- Case II: $n_{p}$ is a participator node. Assume the parent node of $n_{p}$ is $p a\left(n_{p}\right)$. If $n_{p}$ is the left child of $p a\left(n_{p}\right)$, the leftport of $p a\left(n_{p}\right)$ is set to $h$. If $n_{p}$ is the right child of $p a\left(n_{p}\right)$, the rightport of $p a\left(n_{p}\right)$ is set to $h$.

- Case III: $n_{p}$ is a split node. In this case, both the leftport and rightport of $n_{p}$ are set to $h$.

- Case IV: $n_{p}$ is an empty node or a new node. First, we need to check whether $n_{p}$ can be united with its sibling or parent node. If $n_{p}$ can be united, the leftport and rightport of $p a\left(n_{p}\right)$ are updated. In this situation, $p a\left(n_{p}\right)$ becomes a united node, and $n_{p}$ becomes a participator node. Otherwise, $n_{p}$ should be split, and both the leftport and rightport of $n_{p}$ are set to $h$.

Examples: In Figure 4 we show four examples which correspond to the above four announcement cases, respectively. Example 1: [announce 01*:1]. It means to change the next hop of united node E to 1. First we change E's oldport to 1 . Since only the right child of $\mathrm{E}$ is a participate node, we just need to set E's leftport to 1. Example 2: [announce 000*:1]. It means to change the next hop of participator node G to 1. We set G's oldport to 1. Since G is the left child of D, we set D's leftport to 1 . Example 3: [announce $1^{*}: 1$ ]. It means to change the next hop of split node $\mathrm{C}$ to 1 . In this case, we just set C's oldport, leftport and rightport to 1 . Example 4: [announce $\left.0^{*}: 1\right]$. It means to change the next hop of empty node B to 1 . In this case, first we set B's oldport to 1. Then we find that B's sibling node is a split node, thus B and C can be united: first set the leftport and rightport of both B and C to 0, then set A's leftport to 1 , and set A's rightport to 2 .

\subsubsection{Withdrawal Handling}

Given a withdrawal message: [withdraw $p$ ], the node $n_{p}$ corresponding to $p$ must be a prefix node and should be deleted from the trie. First, the oldport 
of $n_{p}$ is set to be empty. Then the leftport and rightport fields are updated according to the node type of $n_{p}$. Specifically, there are three cases as follows.

- Case I: $n_{p}$ is a united node. If both the two child nodes of $n_{p}$ are participator nodes, the leftport and rightport of $n_{p}$ keep unchanged. In this situation, $n_{p}$ is still a united node. If only one of the child of $n_{p}$ is a participator node, it can no longer be united to $n_{p}$. Thus, the child node needs to be split, and $n_{p}$ becomes an empty node: the leftport and rightport of $n_{p}$ are set to be empty. Note that it is possible that the split child node can be united with its child nodes, this unite operation is not performed in our incremental update algorithm in order to guarantee that at most 3 nodes are changed for any update. In this way, the number of prefixes cannot always stay optimal during the update, but the sacrificed compression ratio is negligible. This conclusion is testified by Figure 11. in which the size of the compressed FIB almost keeps unchanged during the one-day update.

- Case II: $n_{p}$ is a participator node. There are two situations: 1) the sibling of $n_{p}$ is also a participator node; 2) the sibling of $n_{p}$ is not a participator node. In the first situation, if the oldport of the parent node $p a\left(n_{p}\right)$ is not empty, the sibling node can be united into $p a\left(n_{p}\right)$; otherwise, the sibling node must be split, and $p a\left(n_{p}\right)$ becomes an empty node. In the second situation, the parent node $p a\left(n_{p}\right)$ must be split. In both situations, $n_{p}$ becomes an empty node.

- Case III: $n_{p}$ is a split node. In this case, both the leftport and rightport of $n_{p}$ are set to be empty, and $n_{p}$ becomes an empty node.

Example: In Figure 4 we show three examples which correspond to the above three withdrawal cases, respectively. Example 1: [withdraw 01*]. It means to delete the united node E. Because E has only one child node I which is a participator node, I can no longer be united to E. Therefore, node I should 325 be split. Specifically, we set E's oldport, leftport, and rightport to 0, and set I's 
leftport and rightport to 7. Example 2: [withdraw 000*]. It means to delete the participator node G. Note that for G's parent node D, we keep its next hop before compression in the variable oldport. Since G's sibling H can still be united into D, we set G's oldport to 0, and set D's leftport to D's oldport 3. Example 3: [withdraw 111*]. It means to delete the split node C. In this case, we set C's oldport, leftport, and rightport to 0.

\subsection{Update Performance Analysis}

For US, when updating a node, at most three nodes need to be changed, while other nodes are not affected. Therefore, the worst case of update time is small bounded. It can be concluded that the update time complexity of US is $O(W)$, where $W$ is the maximum depth of the trie. We compare the update complexity of US with several classic compression algorithms in Table 1. In the sub-trie rooted at the updating node, we use $n$ to represent the number of prefix nodes, use $m$ to represent the number of deleting nodes 4 , and use $c$ to represent the number of distinct next hops. $W$ is 32 for IPv4 FIBs, while $n$ can be pretty large. In the worst case, $n$ is the number of the prefix nodes in the whole trie when the root node is updated. A detailed analysis of update complexity of EAR and ORTC can be found in [16. The 4-level algorithm needs to rebuild the sub-trie rooted at the updating node, thus its time complexity is $\mathrm{O}(\mathrm{n})$. The SMALTA [19] algorithm uses ORTC to take snapshots, thus it has the same compression complexity as ORTC. When inserting or deleting a prefix $N$, SMALTA restores all the compressed nodes in the sub-trie $T_{N}$ rooted at node $N$, so as to correctly perform update. Therefore, many prefix nodes in the sub-trie $T_{N}$ are decompressed, incurring the trie to be not optimal. Although SMALTA only needs to restore the compressed nodes, it needs to judge whether each node in the sub-trie $T_{N}$ is compressed or not. Therefore, it often needs to check all the nodes in the sub-trie $T_{N}$ when updating node $N$. Furthermore, some prefix

\footnotetext{
${ }^{4}$ During the compression process of EAR, ORTC and SMALTA, some leaf nodes are deleted, and they need to be restored during decompression. We call these nodes deleting nodes.
} 
nodes in the sub-trie $T_{N}$ are deleted during compression, thus these deleting nodes need to be re-created during update process. Therefore, the update complexity of SMALTA is $O(m+n)$. We conclude that the update performance of US is the same as that of the original binary trie, and significantly outperforms other compression algorithms.

Table 1: Comparison on time complexities of update.

\begin{tabular}{cc}
\hline \hline Solution & Time complexity \\
\hline Binary trie without compression & $O(W)$ \\
ORTC & $O(c *(m+n))$ \\
EAR & $O(m+n), O(n)$ \\
4-level & $O(n)$ \\
SMALTA & $O(m+n)$ \\
US & $O(W)$ \\
\hline \hline
\end{tabular}

\subsection{US Lookup Algorithm and Complexity Analysis}

The lookup of US abides by the Longest Prefix Matching (LPM) rule 25]. Different from the lookup of the original binary trie, US lookup needs to choose one of the two next hops for each prefix node. Specifically, the lookup of US proceeds in the following steps.

Step I, initialization. Given an incoming IP address $s$, we define a variable $\mathrm{h}$ to store the next hop. Initially, we assign the oldport of the root node to $\mathrm{h}$.

Step II, we obtain the first bit of $s, 1$ ) if it is 1 , we judge whether the rightport of the root node is not empty: if yes, we assign the rightport to h; otherwise, go to step III. 2) if it is 0 , we judge whether the leftport of the root node is not empty: if yes, we assign the leftport to h; otherwise, go to step IV.

Step III, go to the right child node, then obtain the next bit of $s$, and perform the procedure which is similar to step II. If the current node is a leaf node, the algorithm ends. 
Step IV, go to the left child node, then obtain the next bit of $s$, and perform the similar procedure of step II. If the current node is a leaf node, the algorithm ends.

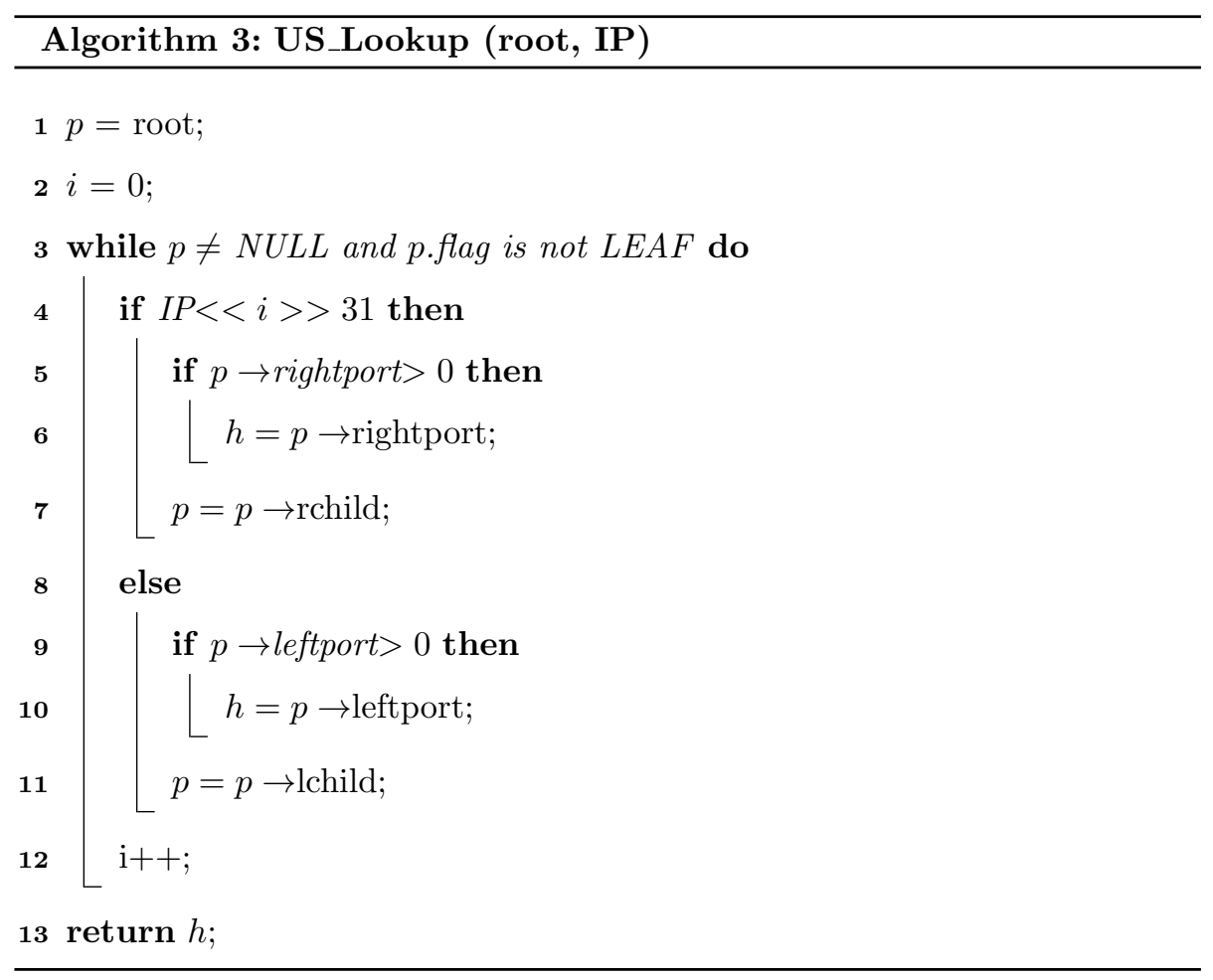
3. In the pseudo codes, root means the root node of the trie, IP means the decimal value of the incoming IP address, and the lookup result is stored in $\mathrm{h}$. Obviously, the time complexity of US lookup in the worst case is $O(W)$, which is the same as that of the original binary trie lookup. Many prefix nodes are united to their parent nodes after US compression, which leads to fewer memory accesses during lookup. Thus, the average lookup speed of US is faster than that of the original binary trie. The simulation results are shown in Section 5.4 


\section{Applications of US}

\subsection{Application to Existing FIB Compression Algorithms}

In practice, US can be combined with many other FIB compression algorithms to achieve further enhanced compression performances. As analyzed above, the update speed of US is fast and the worst case update time is small bounded. Thus, applying US after other compression algorithms will bring little and fixed extra update overhead. Among ORTC, 4_level 22, EAR [16, and auto aggregation [21, ORTC [18] achieves the best compression ratio. Although NSFIB 24] can achieve a better compression ratio than ORTC, the cost is changing the forwarding behavior. Entropy compression 23] pursues to achieve the lower bound of the information entropy, but the compressed result cannot work with prior IP lookup algorithms. US can be applied to the above FIB compression algorithms. Here we apply US to ORTC for the sake of efficiency and practicality. Given a FIB, we first construct a trie, and then compress it using ORTC and get the resulting trie. We further compress the resulting trie using the US algorithm, and get the final compression result. The related simulation results are shown in Section 5.2 .

Here we need to clarify why the compression ratio of ORTC can be further improved. Given the premise that one prefix can only have one next hop and no changes of forwarding behavior happen during compression, ORTC compression is optimal in terms of number of prefix nodes. Given the premise that one prefix can have two next hops, the combination of ORTC and US can achieve a better compression ratio in term of number of prefix nodes. The number of prefixes determines the on-chip memory usage of many IP lookup algorithms, thus we use it as the metric.

\subsection{Application to Classical IP Lookup Solutions}

The US algorithm can also be used to reduce the on-chip memory usage of IP lookup solutions with little additional update overhead. The on-chip memory is usually small, fast and expensive, thus reducing the on-chip memory usage 
can significantly reduce the cost and improve the system efficiency. Generally, IP lookup solutions can be divided into two categories: software based solutions and hardware based solutions. Software based solutions include Lulea [14, LC-trie [26, Tree Bitmap 27], and SAIL [11, etc. Hardware based solutions include using TCAM (such as coolcam [3], parallel TCAMs [5]), using FPGA (such as [7), using both TCAM and FPGA (such as hybrid lookup [28]), and using Bloom filters (such as PBF [29], BF for IPv6 lookups [30]). Among these solutions, we apply the US algorithm to three representative ones: PBF [29], hybrid lookup [28, and SAIL [11. After applying US, the lookup complexity of these fast lookup algorithms keeps unchanged, while the on-chip memory usage is significantly reduced.

Applying US to PBF: for the prefix nodes at each level of the trie, PBF builds one Bloom filter (BF) and one hash table. The BFs are small enough ${ }_{425}$ to be stored in the on-chip memory of FPGA [31, while the hash tables are stored in the off-chip memory. Under a certain false positive probability, there is a positive correlation between the size of a $\mathrm{BF}$ and the number of prefixes inserted into the BF. By applying US compression, the on-chip memory usage of BFs can be reduced because the number of prefixes is reduced. As for the off-chip hash tables, after US compression, each hash bucket stores one prefix and two next hops. Since the number of hash buckets is reduced, the total size of the off-chip hash tables is reduced. The related simulation results are shown in Section 5.5 .

Applying US to hybrid lookup: A trie can be partitioned into two parts the leaf nodes and the trimmed trie. The prefixes of the leaf nodes are stored in TCAM. The trimmed trie is stored in the SRAM-based pipeline of FPGA. After compressing the trie using US, both the number of leaf nodes and the size of the trimmed trie are significantly reduced, thus lead to less memory consumption in both TCAM and FPGA. The related simulation results are shown in Section $440 \quad 5.6$

Applying US to SAIL: SAIL includes four algorithms. SAIL_B is the basic lookup algorithm. For level $i$ of the trie $(0 \leq i \leq 24)$, SAIL_B builds a bit map 
with the length of $2^{i}$, and each prefix node at level $i$ corresponds to a "1" bit in the bit map. SAIL_B stores the bitmaps at level $0 \sim 24$ in the on-chip memory, and thus the upper bound of the on-chip memory usage is $\sum_{i=0}^{24} 2^{i}=2^{25} b i t=4$ MByte. Three optimizations based on SAIL_B in terms of update, lookup, and handling multiple FIBs are SAIL_U, SAIL_L, and SAIL_M, respectively. After using US, most prefix nodes at level 24 are united to level 23, thus we just need to store the bitmaps at level $0 \sim 23$ in the on-chip memory, thus the on-chip memory usage is reduced to a half. The worst case of on-chip memory usage of the four algorithms before and after using US is shown in Table 2 .

Table 2: On-chip memory usage comparsion.

\begin{tabular}{ccccc}
\hline \hline & SAIL_B & SAIL_U $_{\perp}$ & SAIL_L & SAIL_M \\
\hline before using US & $=4 \mathrm{MB}$ & $\leq 2.03 \mathrm{MB}$ & $\leq 2.13 \mathrm{MB}$ & $\leq 2.13 \mathrm{MB}$ \\
after using US & $=2 \mathrm{MB}$ & $\leq 1.016 \mathrm{MB}$ & $\leq 1.07 \mathrm{MB}$ & $\leq 1.07 \mathrm{MB}$ \\
\hline
\end{tabular}

Our algorithm can enhance the cache behavior during IP lookups. For example, assume there are two prefix nodes A and B. As node A and B are often stored separately, the traffic which hits prefix A or B probably does not have good cache behavior. After using our US compression algorithm, node A and $\mathrm{B}$ are compressed into one node, and the next hops of A and B are stored adjacently. Therefore, the traffic which hits A or B will have better cache behavior.

\subsection{Feasibility Analysis}

Legacy routers usually use old TCAMs with small capacity. When the FIB size is close to the TCAM capacity, the TCAM needs to be upgraded when using no compression algorithm. Fortunately, our US algorithm can be used to reduce the memory usage of TCAM. After using our US algorithm, the number of prefixes is compressed to about $65 \%$ of that of the original FIB. In other words, there will be $35 \%$ available memory in the TCAM, and the lifetime of legacy routers can be significantly extended. 
The cost of the US algorithm is that during lookup a second step is needed to obtain the exact next hop for a particular packet, since one prefix is shared by two next hops now. When the US algorithm is applied to hardware routers using TCAMs, we can just use a TCAM chip to output the longest matched prefix and a pointer to the corresponding next hop pair, and use FPGA to conduct the extra logic to choose one next hop. Since FPGA is often used in real routers [32, there is no need to add new hardware for packets lookup. Specifically, we store all the next hop pairs in the SRAM of the FPGA. The output pointer of TCAM points to the corresponding next hop pair in FPGA. FPGA chooses the correct next hop by reading an additional bit of the IP address. The extra logic in FPGA only needs one memory access of the SRAM. The lookup speed of TCAM is slower than that of SRAM [29, 33, 34. As mentioned in [33, the maximum clock rates of SRAM and TCAM are $400 \mathrm{MHz}$ and $266 \mathrm{MHz}$, respectively. That's to say, one memory access in SRAM only needs $2.5 \mathrm{~ns}$, while one clock cycle of TCAM needs 3.76 ns. With pipeline, adding the second step will increase the lookup latency. However, as the second step is faster than looking up TCAM, the bottleneck of system throughput lies in the lookup of TCAM. In other words, adding the second step increases the system latency, but do not affect the system throughput.

${ }_{485}$ As TCAMs are expensive and power-hungry, recent significant work, such as SAIL [1], DXR [35, and Poptrie [12] prefer to use software methods to conduct IP lookup. For the software solutions, it is fairly easy and fast to implement the second step: read one additional bit, and then choose one of the two next hops. Section 5.4 shows that the lookup speed of the compressed trie is faster than that of the original trie, since compression leads to shorter lookup path and better cache performance. Therefore, the US algorithm can be easily applied to software routers, and can also be applied to hardware routers. 


\section{Evaluation}

In this section, we first evaluate the compression performance of US and

495

\subsection{Simulation Setup}

FIBs and Updates: We downloaded 11 BGP FIBs from the RIPE RIS Project [36] at 8:00 AM on September 1st 2014. We name these FIBs as $F I B s_{2014}$. We downloaded 12-year FIBs of rrc00 at 8:005 AM on September 1st from 2003 to 2014, and name these FIBs as FIBs 12 years. We also downloaded one-day update messages for rrc00, rrc01, and rrc03 starting from 8:00 AM September 1st 2014. Table III shows the collecting locations and the sizes of $F I B s_{2014}$.

Synthetic traffic traces: To evaluate the lookup speed of the binary trie before and after US compression, we generate 10M traffic traces based on the simulation FIBs, and guarantee that each prefix is matched with the same probability by the synthetic trace.

Correctness test: We verify the correctness of US by comparing the lookup results of the original FIB and the FIB after US compression using the 4G IPv4 address space. The results are exactly the same, hence our US algorithm passed this correctness test. The appendix contains a proof of the correctness of US using the method proposed in [37].

Computer configuration: We conducted the simulations on a computer with two Intel(R) Core i7-3517U $1.9 \mathrm{GHz} \& 2.4 \mathrm{GHz}$ and $8 \mathrm{~GB}$ RAM running

\footnotetext{
${ }^{5}$ There is no available FIB of rrc00 at 8:00 on September 1st 2004 on the website, thus we use the FIB at 16:00 instead.
} 
Table 3: FIBs used in the simulations.

\begin{tabular}{ccc}
\hline \hline Router ID & Location & \# IPv4 prefixes \\
\hline rrc00 & RIPE NCC, Amsterdam & 532766 \\
rrc01 & LINX, London & 508889 \\
rrc03 & AMS-IX, Amsterdam & 510680 \\
$\operatorname{rrc04}$ & CIXP, Geneva & 512893 \\
rrc05 & VIX, Vienna & 507622 \\
rrc10 & Milan, Italy & 507894 \\
$\operatorname{rrc11}$ & New York, USA & 509738 \\
$\operatorname{rrc12}$ & Frankfurt, Germany & 518739 \\
$\operatorname{rrc13}$ & Moscow, Russia & 552684 \\
$\operatorname{rrc14}$ & Palo Alto, USA & 514408 \\
$\operatorname{rrc15}$ & Sao Paulo, Brazil & 516721 \\
\hline \hline
\end{tabular}

${ }_{520}$ Windows 7 operation system.

\subsection{Simulations on FIB compression}

We evaluate the compression performance using two metrics: compression ratio and compression time. Compression ratio is the ratio between the number of prefixes after compression and that before compression. Smaller compression ratio means more reduction of the FIB size. Compression time is the time used to compress the original FIB. Since ORTC achieves the optimal compression ratio, here we evaluate the compression performance of US using ORTC as a baseline.

\subsubsection{Compression Ratio}

${ }_{530}$ Our simulation results show that US compresses the test FIBs by about 35\%, and improves the compression ratio of ORTC by about $7 \%$ when applied to ORTC. Figure 5 shows the compression ratio changes of US and ORTC on FIB rrc00 over the last 12 years. As time goes by, the FIB size increases rapidly, and the compression ratio of US gets better steadily. In contrast, ORTC 
shows an unstable compression ratio. Figure 6 compares the compression ratio of US and ORTC on FIBs 2014 . US achieves an average compression ratio of 0.65 , which means the compressed FIB size is about $65 \%$ of the original FIB size. The average compression ratio of ORTC is 0.36 . Figure 7 compares the compression ratio of ORTC and ORTC+US on FIBs $s_{2014}$. Results show that combining US and ORTC can reduce the compression ratio of ORTC by about $7 \%$. This does not mean the optimal compression ratio of ORTC is incorrect. It means that the compression ratio can be further improved when one prefix node stores two next hops. In sum, although ORTC achieves smaller compression ratios than US, the compression ratio of ORTC+US is smaller than that of ORTC.

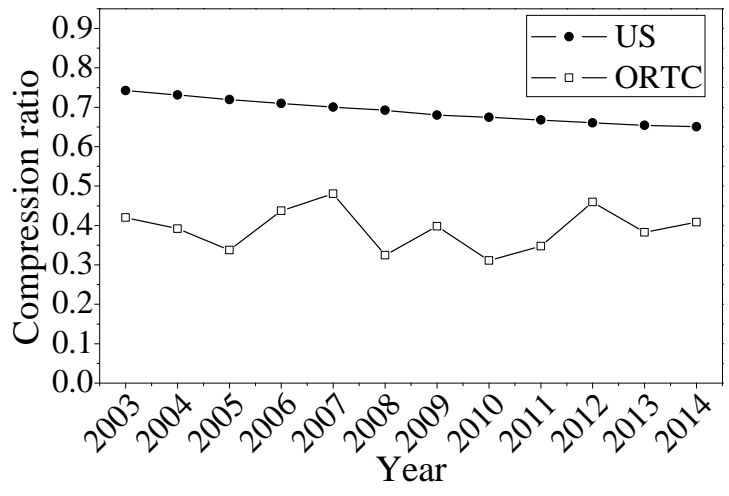

Figure 5: Compression ratios of US and ORTC over the last 12 years.

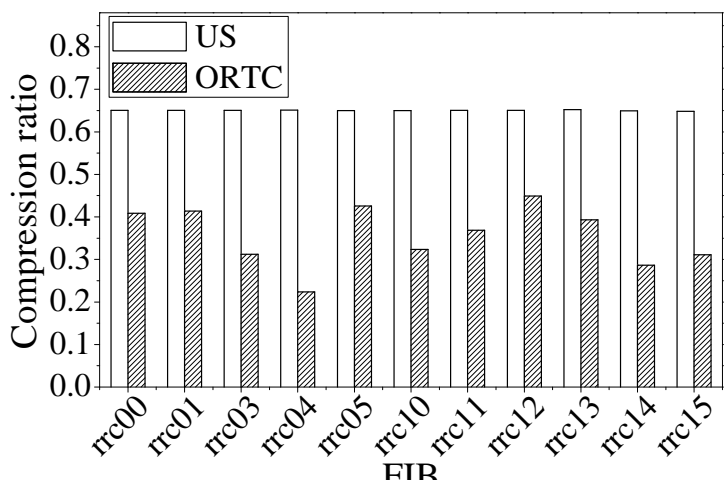

Figure 6: Compression ratios of US and ORTC for 11 FIBs in 2014. 


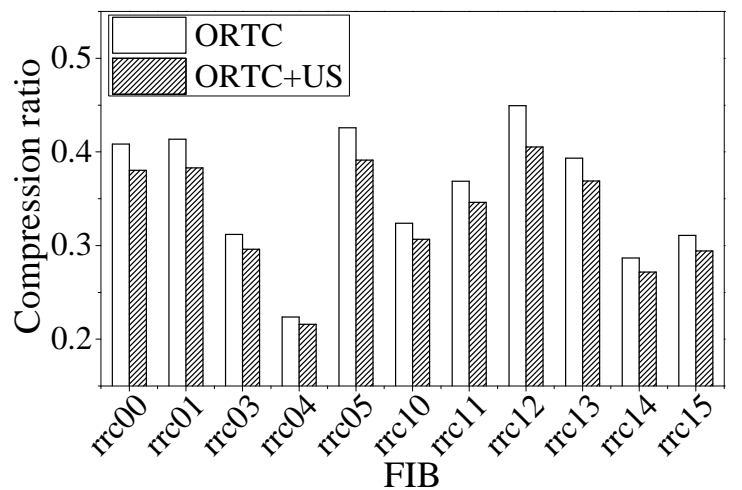

Figure 7: Compression ratios of ORTC and ORTC+US for 11 FIBs in 2014.

\subsubsection{Compression Time}

Our simulation results show that US reduces the compression time by $72 \%$ $77 \%$ comparing to ORTC, and the combination of US and ORTC adds little extra compression time overhead. First, as shown in Figure 8, when using FIB $s_{12 \text { years }}$, the compression time of US is $23 \% \sim 27 \%$ of that of ORTC. As time goes by, the compression time increases with the growing size of FIB, and the increase of the compression time of US is much slower than that of ORTC. Second, as shown in Figure 9, when using FIBs $s_{2014}$, the compression time of US is $25 \% \sim 28 \%$ of that of ORTC. Third, as shown in Figure 10, when using $F I B s_{2014}$, the compression time of ORTC+US is only 1.13 1.16 times of that of ORTC. Note that the compression time of ORTC+US is much less than the sum of ORTC compression time and US compression time. This is because after compressed by ORTC, the trie size is much smaller so that the compression speed of US is faster.

For every trie node, ORTC needs to compute the intersection or union of two next hop sets, and to judge whether a next hop set is a subset of another next hop set. These operations are time-consuming, especially for large FIBs. In contrast, there are only simple assignment and judgment operations in the US compression process. Therefore, the compression speed of US is much faster than that of ORTC.

The US algorithm can be applied to two scenarios. First, for routers with fre- 
quent FIB updates, we can use only US algorithm to achieve a good compression ratio with fast update. Second, for some routers with infrequent FIB updates and limited fast memory, we can use ORTC+US to achieve better compression ratio at the cost of slow update.

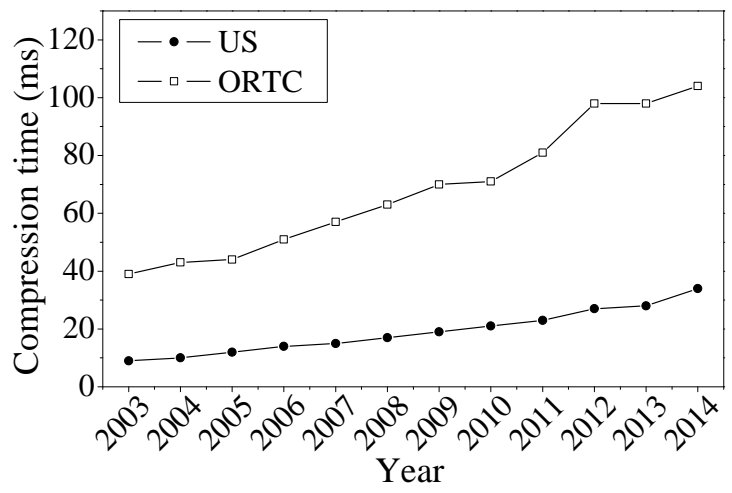

Figure 8: Compression time of US and ORTC over the last 12 years.

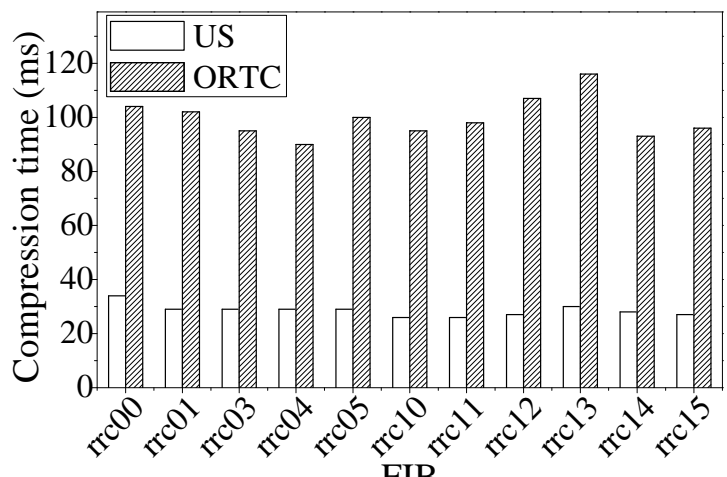

Figure 9: Compression time of US and ORTC for 11 FIBs in 2014.

\subsection{Simulations on Update}

One key advantage of the US algorithm is the fast update with controlled worst case update time. To evaluate the incremental update algorithm of US, we compare the update performance of US with that of the original binary trie, because the update speed of the binary trie without compression is much faster than that of the trie after applying FIB compression algorithms (see Table 1). 


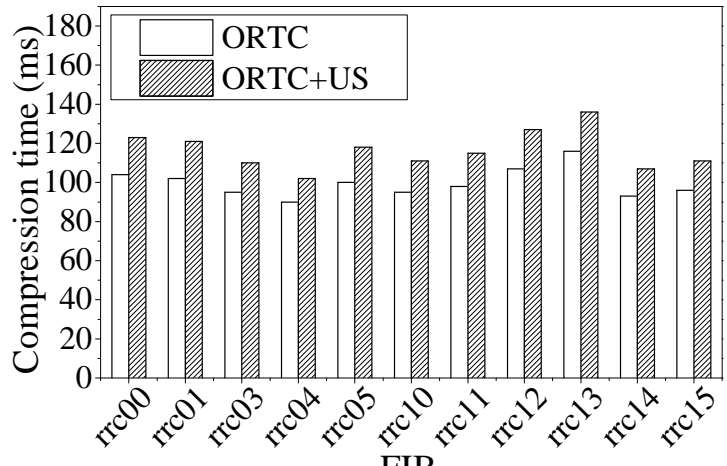

FIB

Figure 10: Compression time of ORTC and ORTC+US for 11 FIBs in 2014.

To evaluate the update overhead of applying US to ORTC, We also compare the update speed of ORTC+US and ORTC in this section. We only show the simulation results for the one-day update of rrc00 which contains 4906067 update messages. The simulation results for $\operatorname{rrc01}$ and $\operatorname{rrc03}$ are similar, thus are omitted due to space limitation.

The update algorithm of ORTC+US works as follows. Given a FIB compressed by ORTC+US and an update message, first we locate the updating node. Then the sub-trie $T$ rooted at the updating node will be de-compressed and updated. Next, we compress the sub-trie $T$ first by ORTC and then by US.

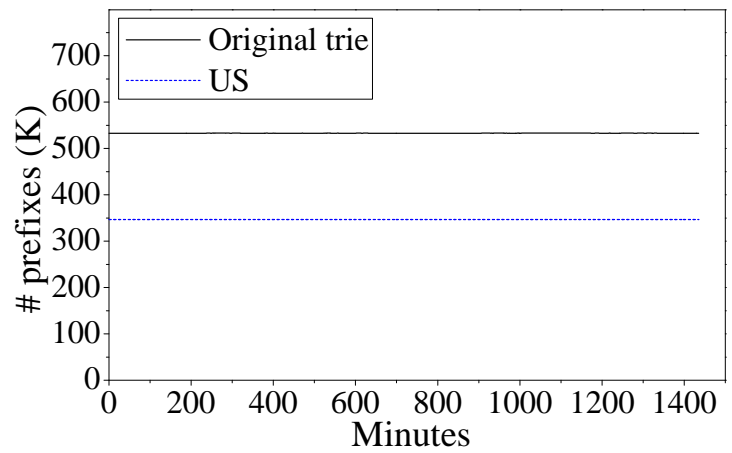

Figure 11: The growth of prefixes for one-day updates.

Our simulation results show that the incremental update of US produces $0.08 \%$ redundant prefix nodes in one day. Figure 11 shows how the number 
of prefixes grows for one-day updates. The x-axes represents the passed min-

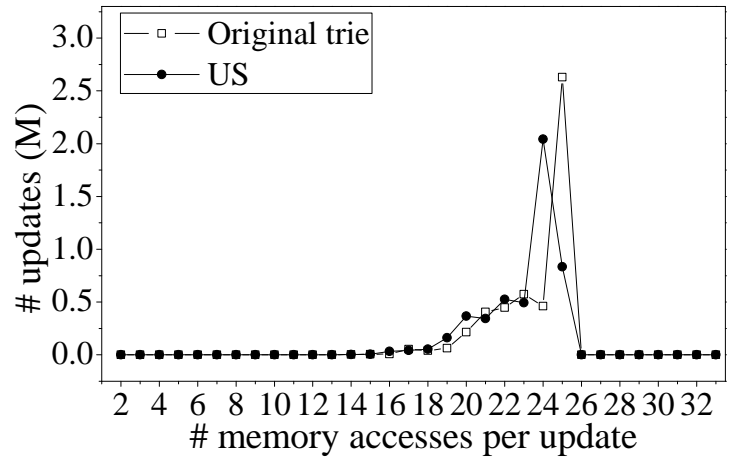

Figure 12: The distribution of the number of memory accesses per update for one-day updates.

Our simulation results show that the update speed of US is a little faster than that of the original binary trie, and much faster than that of ORTC. Figure 13 shows the update speed of the original binary trie, US, ORTC and ORTC+US on one-day update messages. Assume $x$ Mups means $x$ million updates are processed per second. The update speed of the original binary trie ranges from 2.06 Mups to 94.17 Mups with a mean of 18.59 Mups. The update speed of US ranges from 2.16 Mups to 107.75 Mups with a mean of 18.64 Mups. The update 


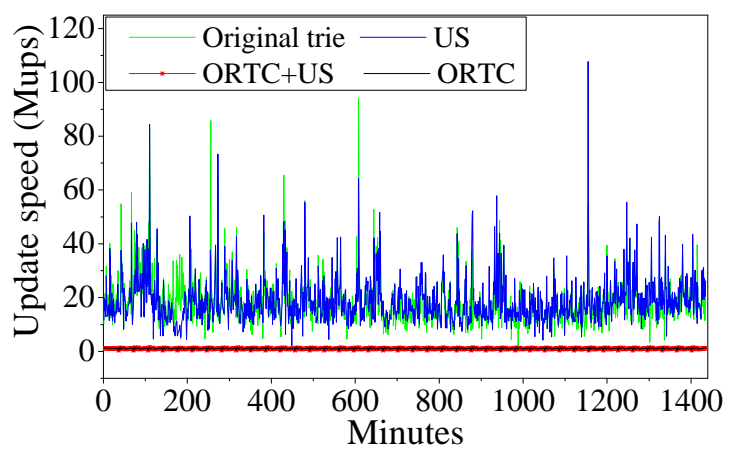

Figure 13: The update speed for one-day updates.

speed of ORTC ranges from 0.35 1.63 Mups with a mean of 1.08 Mups, while the update speed of ORTC+US ranges from 0.33 Mups to 1.60 Mups with a mean of 1.01 Mups. This indicates that when applying US to ORTC, the update speed remains almost unchanged comparing to that of ORTC. In other words, applying US to ORTC can improve the compression ratio with little additional update overhead.

\subsection{Simulations on IP Lookup}

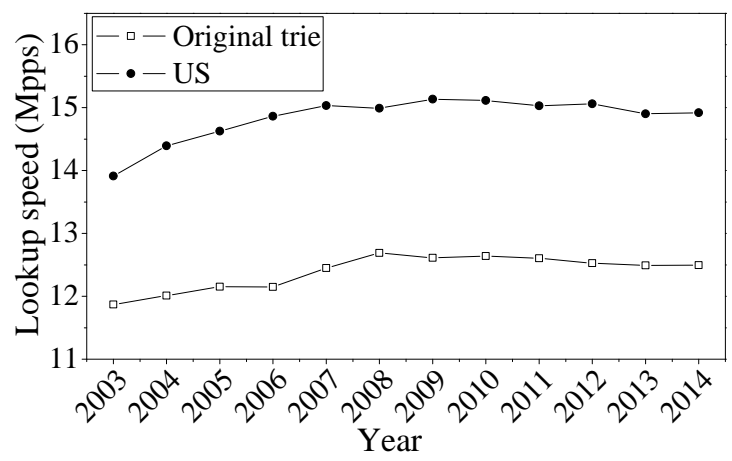

Figure 14: The lookup speed for rrc00 over the last 12 years.

The worst case lookup complexities of the binary trie before and after US compression are both $\mathrm{O}(\mathrm{W})$, where $\mathrm{W}$ is the maximum depth of the binary trie. In practice, after using US, the lookup speed is faster because the average number of memory accesses per lookup is reduced after compression. To verify 


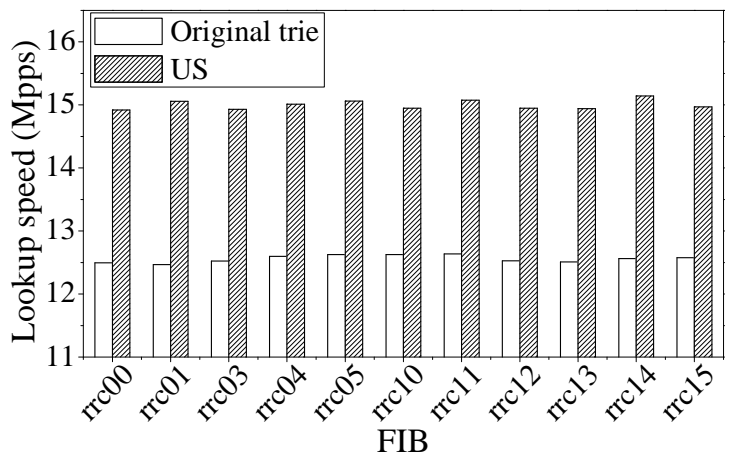

Figure 15: The lookup speed for 11 FIBs in 2014.

this conclusion, we conduct simulations to evaluate the lookup speed of the binary trie before and after using US.

Our simulation results show that the lookup speed of the binary trie is faster after US compression. First, we lookup FIB $B_{12 y e a r s}$ using the corresponding synthetic traffic traces, and the results are shown in Figure 14. The lookup speed after using US ranges from 13.9 to $14.8 \mathrm{Mpps}$ (Million packets per second), which is $1.17 \sim 1.22$ times faster than the lookup speed of the original binary trie. Second, we conduct similar simulations on $F I B_{2014}$, and the results are shown in Figure 15. It shows that the lookup speed after US compression is 1.18 1.21 times faster than that of the original binary trie.

\subsection{Simulations on $P B F$}

In this section, we evaluate the memory usage of PBF before and after using US. We set the number of hash functions of all the Bloom filters to 8, and the sum of the sizes of all the Bloom filters is the on-chip memory usage.

\subsubsection{On-chip memory usage}

Our simulation results show that the on-chip memory usage of PBF is reduced by about 35\% after US compression. The on-chip memory usage for $F I B s_{12 y e a r s}$ is shown in Figure 16. The on-chip memory usage grows year by year because the number of prefixes increases. The on-chip memory usage of PBF before US compression ranges from $1.5 \mathrm{Mb}$ to $6.2 \mathrm{Mb}$. When using 
$\mathrm{US}$, the on-chip memory usage of PBF is reduced to $1.1 \sim 4.0 \mathrm{Mb}$, which is $64.5 \% \sim 73.3 \%$ of the on-chip memory usage before using US. We conduct similar simulations using $F I B s_{2014}$, and the results are shown in Figure 17 . The on-chip memory usage of PBF after using US ranges from 3.81 Mb to 4.16 Mb, and is $64.8 \% \sim 65.2 \%$ of the on-chip memory usage before using US.

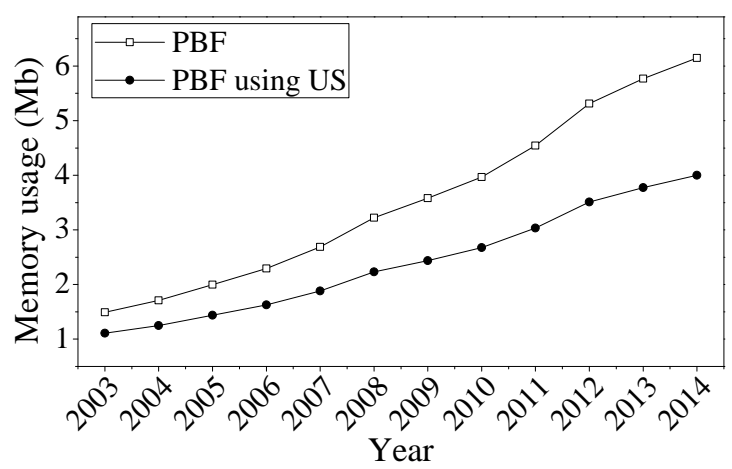

Figure 16: On-chip memory usage of rrc00 over the last 12 years.

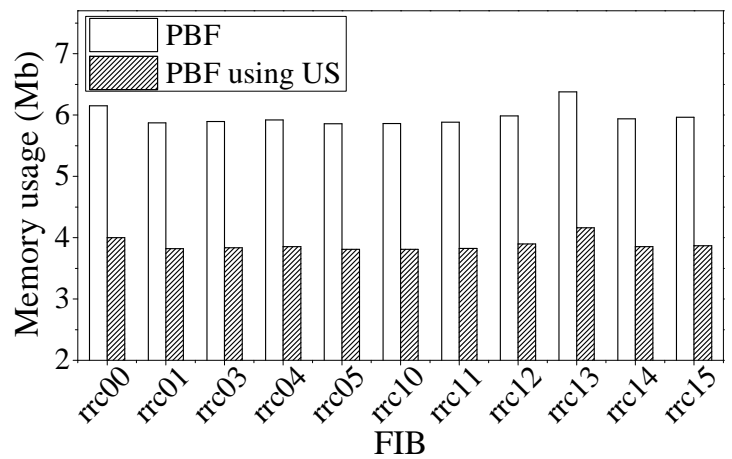

Figure 17: On-chip memory usage for 11 FIBs in 2014.

\subsubsection{Off-chip memory usage}

Our simulation results show that the off-chip memory usage of PBF is reduced by $21.8 \% \sim 22.2 \%$ after US compression. PBF uses hash tables in the off-chip memory. The size of a hash table is proportional to the number of prefixes stored in it. After using US, each hash bucket stores one prefix and two next hops, and the total number of prefixes is reduced. We compare the total 
size of hash tables before and after US compression using $F I B s_{2014}$.

\subsection{Simulations on Hybrid Lookup}

In this section, we evaluate the memory usage of hybrid lookup before and after using US. The update of hybrid lookup includes two parts: updating the

trie structure, and updating TCAM or FPGA. The main advantage of hybrid lookup is the $O(1)$ update complexity when the update of the trie is not considered, because updating the trie do not interrupt lookup. The update complexity is still $O(1)$ when applying US to hybrid lookup, because at most three nodes need to be changed for any update of US. The cost and power consumption of the hybrid lookup algorithm increases linearly with the growth of the number of prefixes stored in FGPA and TCAM. Therefore, we can use US to reduce the number of prefixes while keeping the $O(1)$ update performance.

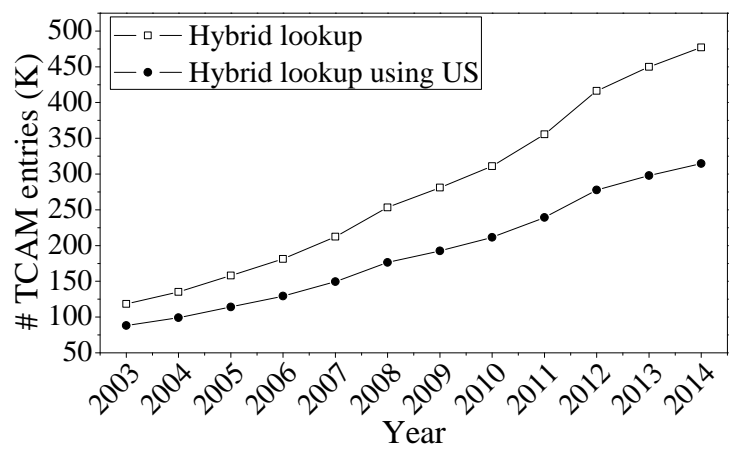

Figure 18: \# prefixes stored in TCAM for rrc00 over the last 12 years.

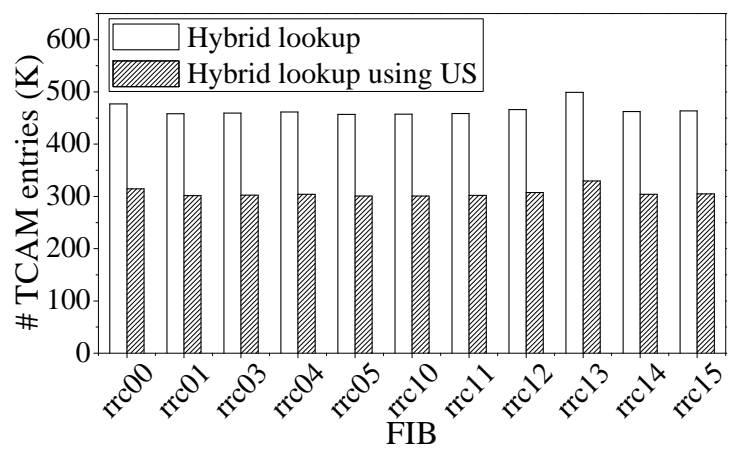

Figure 19: \# prefixes stored in TCAM for 11 FIBs in 2014. 


\subsubsection{TCAM usage}

Our simulation results show that the number of TCAM entries is reduced by of prefixes stored in TCAM), which is also the number of leaf nodes in a trie. Figure 18 shows the simulation results on FIBs 12 years. It can be observed that the number of TCAM entries is reduced to $65 \% \sim 74 \%$ after using US. We conduct similar simulations on $F I B_{2014}$, and the results are shown in Figure 670 19. The number of TCAM entries is reduced to $65 \% \sim 66 \%$ after using US. Note that each prefix (i.e., TCAM entry) corresponds to two next hops, and the next hops are stored in the associated SRAM. Simulation results show that the SRAM usage is $0.45 \sim 0.49 \mathrm{MB}$ before US compression, and becomes $0.64 \sim 0.66 \mathrm{MB}$ after US compression. Such small overhead is negligible.

5.6.2. FPGA usage

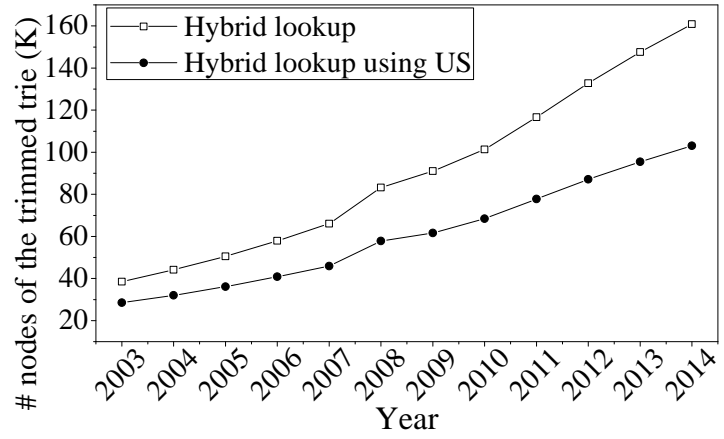

Figure 20: \# trimmed trie nodes stored in FPGA for rrc00 over the last 12 years.

Our simulation results show that the memory usage of FPGA (FPGA usage) is reduced to $71.0 \% \sim 72.0 \%$ after US compression. We evaluate the number of the trimmed trie nodes stored in FPGA. When using FIBs $s_{12 y e a r s}$, as shown in Figure 20, the number of trimmed trie nodes is reduced to $64 \% \sim 74 \%$ after using US. When using $F I B s_{2014}$, as shown in Figure 21, the number of trimmed trie nodes is reduced to $63.9 \% \sim 64.8 \%$. We also evaluate the on-chip memory usage of FPGA for FIBs $s_{2014}$. Simulation results show that the memory usage 


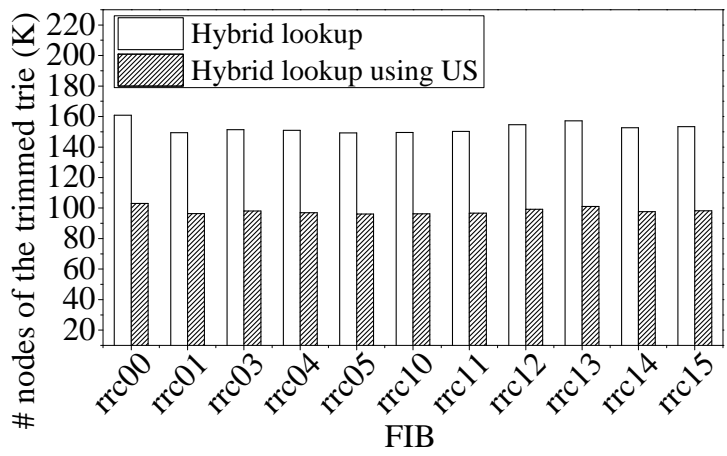

Figure 21: \# trimmed trie nodes stored in FPGA for 11 FIBs in 2014.

of the trimmed trie after US compression is reduced to $71.0 \% \sim 72.0 \%$.

\section{Related Work}

FIB compression is a well studied and important issue due to the significance of FIBs in router design. In this paper, we classify FIB compression algorithms into four categories.

The first category compresses a FIB into a smaller one and does not change the forwarding behavior, such as auto aggregation [21, ORTC [18, EAR [16], and wild-card compression [38]. The auto aggregation algorithm [21] only compresses the sibling prefix nodes with the same next hop. This compression algorithm is simple and fast, but its compression ratio is not attractive and the update time is not small bounded. ORTC [18] achieves the theoretical optimal compression ratio in terms of the number of prefixes. It traverses the trie three passes to complete the compression. Generally speaking, the update of one prefix can be implemented by re-constructing the sub-trie rooted at the updating trie node using the same compression algorithm. However, optimal compression is complicated and slow, thus leads to complicated and slow update. The authors in [18] did not present the update algorithm of ORTC. To address the update problem, Liu et al. proposed two incremental update algorithms in 20] for ORTC, and also proposed FIFA-S, FIFA-A and FIFA-H in [17] to further improve the update performance. Uzmi et al. [19] also proposed an 
update algorithm for ORTC. The main idea is to only update the nodes which are affected, so as to accelerate the update speed. The key reason of slow update is due to optimal compression. Thus sub-optimal compression with fast update becomes an appealing alternative choice. Yang et al. proposed two sub-optimal compression algorithms, EAR_slow and EAR_fast [16]. Compared with ORTC, EAR_fast can reduce the compression time to around $1 / 10$ at the cost of a little compression ratio loss. Although EAR_fast can achieve fast update speed, the worst case update time is still not small bounded. LFA [39] and BLOCK 40. optimize the update performance by leveraging the temporal and spatial locality of udpates. The basic idea of these two algorithms is similar: only the sub-tries which are not updated for a preset period of time are compressed. If an update occurs in a compressed sub-trie, the compressed sub-trie will be forced to be de-compressed/split and then be updated. For the update complexity, the best case of these two algorithms is $O(W)$, and the worst case is $O(n)$, where $W$ is the maximum depth of the trie, and $n$ is the number of nodes in the subtrie. Different from above compression methods based on binary trie, wild-card compression [38] compresses the prefixes into new ones, and each bit has three states: 0,1 , and don't care. Thus it can only be applied to TCAM-based IP lookup solutions. What is more, its update is really complicated and slow. In summary, the worst case update of all the above FIB compression and update algorithms is to update the whole sub-trie rooted at the updating node. US also belongs to this category, but only needs to change at most three nodes in the worst case of update. The update complexity of US is always $O(W)$.

The second category aims at achieving a better compression ratio at the cost of changing the forwarding behavior, such as NSFIB 24] and 4-level 22]. NSFIB can achieve a much better compression ratio than ORTC by taking advantage of multiple next hops. The 4-level algorithm defines four levels of FIB compression. 730 The first two levels are simple, but the compression is not sufficient. The last two levels achieve better compression ratios at the cost of forwarding some packets which should have been dropped.

The third category focuses on compressing the FIB towards the information 
entropy bounds, and the compressed result is represented by bits rather than prefixes. Rétvári et al. [23] introduced the information entropy of tries for the first time, and there are two successors. Rottenstreich et al. 41. proposed an encoding scheme to achieve sub-optimal memory requirement, and Korosi et al. 42 focused on improving the lookup speed. The common disadvantage of this category is the complicated and slow update performance.

The above three categories are purely local solutions, and do not affect neighboring routers. The fourth category requires the coordination between routers or between switches and controllers. In [43], three route aggregation strategies are proposed to compress the FIBs. These strategies either require coordination between the ASes or need to change the way routers build their FIBs. A recent work 44 focuses on minimizing the number of updates sent from the controller to the compressed FIBs stored in switches.

\section{Conclusion}

With the rapid growth of FIB size in backbone routers, FIB compression becomes a hot topic in recent years, and various FIB compression algorithms have been proposed. The update performance will inevitably be degraded if a FIB is compressed. Only when the worst case of update time is small bounded, the risk of packet loss during updates can ultimately be avoided. Towards this goal, we propose the Unite and Split (US) compression algorithm in this paper to achieve fast update with small bounded worst case (i.e., at most three nodes need to be changed per update). Further, we use the US algorithm to improve the performance of several classic software and hardware lookup algorithms. Simulation results on real-world FIBs show that the compression ratio of US is about $65 \%$ with fast compression time (only about $28.5 \mathrm{~ms}$ ), and the update speed of US is fast. In addition, the on-chip memory usage of several classic lookup algorithms is significantly reduced after applying US. To enable others to replicate the simulations, we released the source code of our US algorithm and related data set at Github [45]. 


\section{Acknowledgements}

The research is supported by the National Basic Research Program of Chi-

Foundation of China under Grant 61133015, and Specialized Research Fund for the Doctoral Program of Higher Education under Grant 20120002110060.

\section{References}

[1] BGP routing table analysis reports, http://bgp.potaroo.net/.

[2] 512k bug [on line], Available: http://www.theinquirer.net/inquirer/news/2360306/512krouting-bug-might-kill-the-internet-but-probably-wont.

[3] F. Zane, G. Narlikar, A. Basu, Coolcams: Power-efficient TCAMs for forwarding engines, in: Proc. IEEE INFOCOM, Vol. 1, IEEE, 2003, pp. 42-52.

[4] T. Yang, R. Duan, J. Lu, S. Zhang, H. Dai, B. Liu, Clue: achieving fast update over compressed table for parallel lookup with reduced dynamic redundancy, in: Proc. IEEE ICDCS, 2012, pp. 678-687.

[5] K. Zheng, C. Hu, H. Lu, B. Liu, A TCAM-based distributed parallel IP lookup scheme and performance analysis, Networking, IEEE/ACM Transactions on 14 (4) (2006) 863-875.

[6] V. Ravikumar, R. N. Mahapatra, L. N. Bhuyan, Easecam: An energy and storage efficient tcam-based router architecture for ip lookup, Computers, IEEE Transactions on 54 (5) (2005) 521-533.

[7] H. Le, W. Jiang, V. K. Prasanna, A SRAM-based architecture for trie-based IP lookup using FPGA, in: Proc. IEEE FCCM, IEEE, 2008, pp. 33-42.

[8] H. Le, W. Jiang, V. K. Prasanna, A sram-based architecture for trie-based ip lookup using fpga, in: Field-Programmable Custom Computing Machines, 2008. FCCM'08. 16th International Symposium on, IEEE, 2008, pp. $33-42$. 
[9] H. Le, V. K. Prasanna, Scalable high throughput and power efficient iplookup on fpga, in: Field Programmable Custom Computing Machines, 2009. FCCM'09. 17th IEEE Symposium on, IEEE, 2009, pp. 167-174.

[10] W. Jiang, V. K. Prasanna, A memory-balanced linear pipeline architecture for trie-based ip lookup, in: High-Performance Interconnects, 2007. HOTI 2007. 15th Annual IEEE Symposium on, IEEE, 2007, pp. 83-90.

[11] T. Yang, G. Xie, Y. Li, Q. Fu, A. X. Liu, Q. Li, L. Mathy, Guarantee IP lookup performance with FIB explosion, in: Proc. ACM SIGCOMM, 2014, pp. 39-50.

[12] H. Asai, Y. Ohara, Poptrie: A compressed trie with population count for fast and scalable software IP routing table lookup, in: Proc. ACM SIGCOMM, 2015.

[13] S. Nilsson, G. Karlsson, Ip-address lookup using lc-tries, Selected Areas in Communications, IEEE journal on 17 (6) (1999) 1083-1092.

[14] M. Degermark, A. Brodnik, S. Carlsson, S. Pink, Small forwarding tables for fast routing lookups, Vol. 27, ACM, 1997.

[15] T. Yang, Z. Mi, R. Duan, X. Guo, J. Lu, S. Zhang, X. Sun, B. Liu, An ultrafast universal incremental update algorithm for trie-based routing lookup, in: Proc. IEEE/ACM ICNP, 2012.

[16] T. Yang, B. Yuan, S. Zhang, T. Zhang, R. Duan, Y. Wang, B. Liu, Approaching optimal compression with fast update for large scale routing tables, in: Proc. IEEE IWQoS, 2012, p. 32.

[17] Y. Liu, B. Zhang, L. Wang, FIFA: Fast incremental FIB aggregation, in: Proc. IEEE INFOCOM, 2013.

[18] R. P. Draves, C. King, S. Venkatachary, B. D. Zill, Constructing optimal IP routing tables, in: Proc. IEEE INFOCOM, 1999. 
[19] Z. A. Uzmi, M. Nebel, A. Tariq, S. Jawad, R. Chen, A. Shaikh, J. Wang, P. Francis, SMALTA: practical and near-optimal FIB aggregation, in: Proc. ACM CoNEXT, ACM, 2011, p. 29.

[20] Y. Liu, X. Zhao, K. Nam, L. Wang, B. Zhang, Incremental forwarding table aggregation, in: Proc. IEEE GLOBECOM, 2010.

[21] B. Cain, Auto aggregation method for IP prefix/length pairs, uS Patent 6,401,130 (Jun. 4 2002).

[22] X. Zhao, Y. Liu, L. Wang, B. Zhang, On the aggregatability of router forwarding tables, in: Proc. IEEE INFOCOM, 2010.

[23] G. Rétvári, J. Tapolcai, A. Kőrösi, A. Majdán, Z. Heszberger, Compressing IP forwarding tables: Towards entropy bounds and beyond, in: Proc. ACM SIGCOMM, 2013.

[24] Q. Li, D. Wang, M. Xu, J. Yang, On the scalability of router forwarding tables: Nexthop-selectable FIB aggregation, in: Proc. IEEE INFOCOM, IEEE, 2011, pp. 321-325.

[25] M. A. Ruiz-Sanchez, E. W. Biersack, W. Dabbous, Survey and taxonomy of IP address lookup algorithms, Network, IEEE 15 (2) (2001) 8-23.

[26] S. Nilsson, G. Karlsson, IP-address lookup using LC-tries, Selected Areas in Communications, IEEE Journal on 17 (6) (1999) 1083-1092.

[27] W. Eatherton, G. Varghese, Z. Dittia, Tree bitmap: hardware/software IP lookups with incremental updates, ACM SIGCOMM Computer Communication Review 34 (2) (2004) 97-122.

[28] L. Luo, G. Xie, Y. Xie, L. Mathy, K. Salamatian, A hybrid IP lookup architecture with fast updates, in: Proc. IEEE INFOCOM, 2012.

[29] S. Dharmapurikar, P. Krishnamurthy, D. E. Taylor, Longest prefix matching using bloom filters, in: Proc. ACM SIGCOMM, 2003. 
[30] H. Song, F. Hao, M. Kodialam, T. Lakshman, IPv6 lookups using distributed and load balanced bloom filters for 100gbps core router line cards, in: Proc. IEEE INFOCOM, IEEE, 2009, pp. 2518-2526.

[31] FPGA data sheet [on line], Available: http://www.xilinx.com.

845 [32] Cisco website, http://www.cisco.com/c/en/us/td/docs/routers/ asr1000/install/guide/asr1routers/asr1higV8/asr1_hw2.html.

[33] H. Le, W. Jiang, V. K. Prasanna, Scalable high-throughput sram-based architecture for ip-lookup using fpga, in: Field Programmable Logic and Applications, IEEE, 2008, pp. 137-142.

[34] D. Kreutz, F. M. V. Ramos, P. Verissimo, C. E. Rothenberg, S. Azodolmolky, S. Uhlig, Software-defined networking: A comprehensive survey, Proceedings of the IEEE 103 (1) (2015) 14-76.

[35] M. Zec, L. Rizzo, M. Mikuc, Dxr: towards a billion routing lookups per second in software, ACM SIGCOMM Computer Communication Review 42 (5) (2012) 29-36.

[36] RIPE network coordination centre [on line], Available: http://www.ripe.net/data-tools/stats/ris/ris-raw-data.

[37] T. Yang, G. Xie, et al., A fresh look at forwarding information base compression via mathematical analysis, in: Network Operations and Management Symposium (NOMS), IEEE, 2014, pp. 1-4.

[38] H. Yu, A memory-and time-efficient on-chip TCAM minimizer for IP lookup, in: Proc. IEEE DATE, 2010.

[39] N. Sarrar, R. Wuttke, S. Schmid, M. Bienkowski, S. Uhlig, Leveraging locality for fib aggregation, in: Global Communications Conference (GLOBECOM), 2014 IEEE, IEEE, 2014, pp. 1930-1935. 
[40] M. Bienkowski, S. Schmid, Competitive fib aggregation for independent prefixes: Online ski rental on the trie, in: Structural Information and Communication Complexity, Springer, 2013, pp. 92-103.

[41] O. Rottenstreich, M. Radan, Y. Cassuto, I. Keslassy, C. Arad, T. Mizrahi, Y. Revah, A. Hassidim, Compressing forwarding tables, in: Proc. IEEE INFOCOM, IEEE, 2013, pp. 1231-1239.

[42] A. Korosi, J. Tapolcai, B. Mihálka, G. Mészáros, G. Rétvári, Compressing IP forwarding tables: Realizing information-theoretical space bounds and fast lookups simultaneously, in: IEEE ICNP, 2014.

[43] J. L. Sobrinho, F. Le, A fresh look at inter-domain route aggregation, in: Proc. IEEE INFOCOM, IEEE, 2012, pp. 2556-2560.

[44] M. Bienkowski, N. Sarrar, S. Schmid, S. Uhlig, Competitive FIB aggregation without update churn, in: Proc. IEEE ICDCS, 2014.

[45] Source code of US [on line], Available: https://github.com/ussource.

\section{Appendix}

The authors of [37] proposed a universal method to prove the correctness of FIB compression algorithms. We use this method to prove the correctness of the US algorithm below. First, as mentioned in [37, the prefixes are represented by regular expression syntax, and the symbols used are defined as follows.

- $q$ is a node in the trie, and $(q)$ represents the corresponding prefix of node $q$. Prefix nodes have next-hops, while empty nodes don't.

- $\left(q_{1} q_{2}\right)$ represents the bit string of the path between prefix nodes $q_{1}$ and $q_{2}$, where no prefix nodes appear in the path.

- $L(q)$ represents the prefix length of node $q$. hop of prefix $(q)$ in trie $P$. 
- $(\bar{q})$ represents a prefix with the same length of $(q)$, but it is different from $(q) . P(\bar{q})$ means the next hop of prefix $(\bar{q})$ in trie $P$.

- $(a \mid b)$ represents a prefix with two next hops. Its leftport is $a$, and rightport is $b$. Given one more bit 0 or 1 , there is $(a \mid b)_{0}=a$ and $(a \mid b)_{1}=b$.

The operation of XOR is defined as follows:

$$
\begin{gathered}
\forall x, y \in G \\
x \oplus y=\left\{\begin{array}{lr}
x+y, & x y(x+y)=0 \\
y, & x>0, y>0 \\
\text { meaningless, } & \text { otherwise }
\end{array}\right.
\end{gathered}
$$

$\forall$ IP address $\mathrm{R}, \mathrm{R}=[0,1]\{32\}$, suppose the match result of the most significant $i$ bits is $S_{i}$, then the next-hop of $\mathrm{R}$ is $P(R)=S_{1} \oplus S_{2} \oplus \ldots S_{32}=\oplus_{i=1}^{32} S_{i}$. 22 of our US algorithm. The proof of other models is similar.

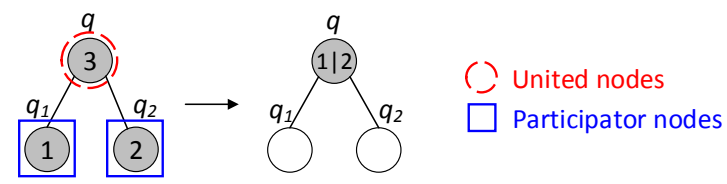

Figure 22: The first model of US.

Proof. $\forall$ IP address R, obviously, $L(R)=K, R=[0,1] K$. Suppose $R=[0,1] L(q)[0,1][0,1]\{K-$ $L(q)-1\}$.

Step1: matching $[0,1]\{L(q)\}$

$$
[0,1]\{L(q)\}=(q) \Rightarrow\left\{\begin{array}{l}
P 1([0,1]\{L(q)\})=3 \\
P 2([0,1]\{L(q)\})=(1 \mid 2)
\end{array}\right.
$$

$$
\left.\begin{array}{c}
{[0,1]\{L(q)\} \neq(q) \Rightarrow P 1=P 2} \\
P 1(\bar{q})=P 2(\bar{q})
\end{array}\right\} \Rightarrow \text { Only considering }[0,1]\{L(q)\}=
$$

$(q)$ 
Step2: when $[0,1]\{\mathrm{L}(\mathrm{q})\}=(\mathrm{q})$, matching $[0,1]$

$$
[0,1]=0, \text { or } 1 \Rightarrow\left\{\begin{array}{r}
P 1(0)=P 1\left(q_{1}\right)=3 \oplus 1=1 \\
P 1(1)=P 1\left(q_{2}\right)=3 \oplus 2=2 \\
P 2(0)=(1 \mid 2)_{0}=1 \\
P 2(1)=(1 \mid 2)_{1}=2
\end{array}\right\}
$$

$\Rightarrow P 1([0,1])=P 2([0,1])$

At this stage,

$P 1([0,1]\{L(q)\})[0,1])=P 1([0,1]\{L(q)\})) \oplus P 1([0,1])=P 1([0,1])$

$P 2([0,1]\{L(q)\})[0,1])=P 2([0,1]\{L(q)\})) \oplus P 2([0,1])=P 2([0,1])$

Thus, $P 1([0,1]\{L(q)\})[0,1])=P 2([0,1]\{L(q)\})[0,1])$.

For P1 and P2, they have the same rest parts. In other words, when matching

According to step1 and step2, $P 1 \Leftrightarrow P 2$. 